\title{
ON THE ISOMORPHISM PROBLEM FOR LIE ALGEBRAS
}

\section{J. KNOPFMACHER}

Given presentations $P, P^{\prime}$ of two algebraic systems $L$ and $L^{\prime}$ in terms of finitely many generators and relations, the isomorphism problem asks if it can be determined from $P$ and $P^{\prime}$ whether or not $L$ is isomorphic to $L^{\prime}$. The main purpose of this note is to discuss some invariants of the isomorphism type of a Lie algebra $L$, which may be derived from any finite presentation of $L$, and easily used to distinguish between many different nonisomorphic algebras.

The invariants are analogous to the elementary ideals and knot polynomials used in studying certain finitely-presented groups (cf. [3]). They may be derived by associating (in a slightly unusual way) with finitely-generated presentations of an associative algebra $A$, certain ideals in its largest commutative quotient $A_{c}$. These ideals depend only on the isomorphism type of $A$, and are particularly amenable when $A_{c}$ is a polynomial ring, a situation which frequently arises when $A$ is the universal enveloping algebra of some Lie algebra $L$.

They may also be applied to special Jordan algebras, and, of course, to word problems for algebras.

I am indebted to the referee for a suggestion concerning the form of this paper.

1. Associative presentations. All algebras will be over a fixed commutative ring $K$ with identity. Let $\Phi$ be a free associative, but noncommutative, algebra with identity on indeterminates $x_{1}, \cdots, x_{n}$. Define the free semiderivatives $D_{i}=D_{x_{i}}: \Phi \rightarrow \Phi$ to be the linear maps given inductively by:

$$
D_{i}\left(x_{j}\right)=\delta_{i j} \text { and } \quad D_{i}(u i)=D_{i}(u) \cdot v .
$$

(A similar discussion could be developed in terms of the usual partial derivatives, but this would yield trivial information when applied to Lie algebras.)

Now let $A$ be an associative algebra with a presentation $P=\left(x_{1}, \cdots, x_{n} / r_{1}, r_{2}, \cdots\right)$ as a quotient of $\Phi$ by the ideal generated by elements $r_{1}, r_{2}, \cdots$, under an epimorphism $\pi: \Phi \rightarrow A$. Let $\alpha: A \rightarrow A$ 。 denote the natural epimorphism of $A$ onto its largest commutative quotient $A_{c}=A /[A, A]$. Consider the sequence of maps

Received by the editors September 10, 1966. 


$$
\Phi \stackrel{D_{i}}{\rightarrow} \Phi \stackrel{\pi}{\rightarrow} A \stackrel{\alpha}{\rightarrow} A_{c} .
$$

Algebraic techniques of Fox in knot theory suggest the following:

Definition 1.1. The Alexander-Fox matrix of $P$ is the matrix $A(P)$ $=\left(a_{i j}\right)$ over $A_{c}$ defined by $a_{i j}=\alpha \pi D_{j}\left(r_{i}\right)$.

Referring to [3], say, for the definition of the elementary ideals of a matrix over a commutative ring, consider

Definition 1.2. The kth elementary ideal of $P$ in $A_{c}$ is the corresponding ideal $E_{k}(P)$ of $A(P)[k=0,1,2, \ldots]$.

THEOREM 1.3. If $P$ and $Q$ are any two finitely-generated presentations of $A$, then (for each $k$ ) $E_{k}(P)=E_{k}(Q)=E_{k}(A)$, say.

Proof. By the Tietze-Shoda theorem on presentations (cf. [2], Chapter III), it is sufficient to show that the ideals are invariant under "Tietze operations" on the previous presentation $P$. To do this, first note that every element $s$ of the ideal of $\Phi$ generated by $r_{1}, r_{2}, \cdots$ is expressible as a $K$-linear combination of monomials of the form $w=a r_{i} b$ or $r_{i} b(a, b \in \Phi)$; hence $D_{j}(w)=0$ or $\pi D_{j}\left(r_{i}\right) \cdot \pi b$. Thus adjunction of $s$ to $P$ gives rise to a matrix "equivalent" to $A(P)$ (cf. [3]). Finally, suppose that a new indeterminate $x_{n+1}$ and a new relator $x_{n+1}-u(u \in \Phi)$ is adjoined to $P$. Then the Alexander-Fox matrix of the new presentation has the form

$$
\left(\begin{array}{cc}
A(P) & 0 \\
\cdots & 1
\end{array}\right)
$$

which is also "equivalent" to $A(P)$.

If $f: A \rightarrow A^{\prime}$ is an associative algebra isomorphism, it may be noted that (for each $k$ ) $E_{k}(A) \cong E_{k}\left(A^{\prime}\right)$ under the induced isomorphism $f_{*}: A_{c} \rightarrow A_{c}^{\prime}$. Ideals may also be defined for presentations of commutative associative algebras as quotients of polynomial rings.

Now suppose that an associative algebra $A$ be called "nice" if it is finitely presentable, $K$ is a unique factorization domain, and $A_{c}$ is a polynomial ring over $K$.

Definition 1.4. The kth invariant polynomial $\Delta_{k}=\Delta_{k}(A)$ of a "nice" algebra $A$ is (ambiguously) any generator of the smallest principal ideal of $A_{c}$ which contains $E_{k}(A)[k=0,1,2, \ldots]$.

Thus if $A$ has a finite presentation $P=\left(x_{1}, \cdots, x_{n} / r_{1}, \cdots, r_{m}\right)$, then $\Delta_{k}=1$ for $k \geqq n, \Delta_{k}=0$ for $k<n-m$, and, for $0<n-k \leqq m, \Delta_{k}$ is the g.c.d. of the determinants of all $(n-k)$-rowed minors of $A(P)$ (cf. [3]). Since elementary ideals are ascending, $\Delta_{k+1} \mid \Delta_{k}$ for $k=0,1,2, \cdots$.

Corollary 1.5. If $A$ and $A$ ' are "nice" associative algebras and 
$A \cong A^{\prime}$, then (for each $\left.k\right) \Delta_{k}(A)=\Delta_{k}\left(A^{\prime}\right)$ essentially.

2. Lie algebras. Let $F$ be a free Lie algebra on free generators $x_{1}, \cdots, x_{n}$, and let $L$ be a Lie algebra with a presentation $P$ $=\left(x_{1}, \cdots, x_{n} / r_{1}, r_{2}, \cdots\right)$ as a quotient of $F$ by the ideal generated by elements $r_{1}, r_{2}, \cdots$, under an epimorphism $\pi: F \rightarrow L$. Let $\alpha: L \rightarrow L$. denote the natural epimorphism of $L$ on to its largest zero algebra quotient $L_{o}=L / L^{2}$. If $U(R)$ denotes the universal enveloping algebra of a Lie algebra $R$, then $\pi$ and $\alpha$ induce epimorphisms

$$
\pi_{*}: U(F) \rightarrow U(L) \text { and } \alpha_{*}: U(L) \rightarrow U\left(L_{\bullet}\right) .
$$

Further, if $A=U(L)$, then $\pi_{*}$ defines a presentation $P_{*}$ of $A$ as a quotient of the free associative algebra $\Phi=U(F)$, and $\alpha_{*}$ coincides with the natural epimorphism $A \rightarrow A_{c}$.

It follows that we may associate with $P$ the Alexander-Fox matrix $A\left(P_{*}\right)$ over $U\left(L_{0}\right)$, and that this leads to elementary ideals $E_{k}(L)$ depending only on the isomorphism type of $L$. In addition, if the Lie algebra $L$ is "nice", i.e. it is finitely presentable, $K$ is a unique factorization domain, and $L_{0}$ is a free $K$-module, then $A=U(L)$ is a "nice" associative algebra. Hence in this case we may associate with $L$ invariant polynomials $\Delta_{k}(L)$ depending only on the isomorphism type of $L$.

EXAMPLE 2.1. Suppose that $L$ has the presentation

$$
\begin{aligned}
P=\left(x_{1}, x_{2}, x_{3} /\left[\left[x_{1}, x_{2}\right],\left[x_{1}, x_{3}\right]\right]+\right. & {\left[\left[\left[x_{2}, x_{3}\right], x_{2}\right], x_{1}\right], } \\
& {\left.\left[\left[\left[x_{1}, x_{2}\right], x_{2}\right], x_{3}\right]+\left[x_{2},\left[x_{3}, x_{1}\right]\right]\right) }
\end{aligned}
$$

and that $L^{\prime}$ has the presentation

$$
\begin{array}{r}
P^{\prime}=\left(x_{1}, x_{2}, x_{3} /\left[x_{1},\left[\left[x_{2}, x_{3}\right], x_{3}\right]\right]+\left[x_{1},\left[x_{1}, x_{2}\right]\right],\right. \\
\left.\left[\left[\left[x_{2},\left[x_{3}, x_{1}\right]\right], x_{3}\right], x_{2}\right]+\left[\left[\left[x_{2}, x_{1}\right], x_{3}\right], x_{3}\right]\right) .
\end{array}
$$

Then $L_{\bullet}$ and $L_{\bullet}^{\prime}$ are both isomorphic to a $K$-module with free $K$ basis $\left\{t_{1}, t_{2}, t_{3}\right\}$. One has

$$
\begin{aligned}
& A\left(P_{*}\right)=\left(\begin{array}{crr}
0 & t_{1} t_{2} t_{3} & -t_{1} t_{2}^{2} \\
t_{2} t_{3}\left(1+t_{2}\right) & -t_{1} t_{2} t_{3} & -t_{1} t_{2}
\end{array}\right) \text { and } \\
& A\left(P_{*}^{\prime}\right)=\left(\begin{array}{ccc}
-t_{1} t_{2} & t_{1}\left(t_{1}-t_{3}^{2}\right) & t_{1} t_{2} t_{3} \\
t_{2} t_{3}^{2}\left(t_{2}-1\right) & t_{1} t_{3}^{2} & -t_{1} t_{2}^{2} t_{3}
\end{array}\right) .
\end{aligned}
$$

Hence

$$
\begin{aligned}
\Delta_{1}(L) & =t_{1} t_{2}^{2} t_{3}\left(1+t_{2}\right), \quad \Delta_{2}(L)=t_{2}, \\
\Delta_{1}\left(L^{\prime}\right) & =t_{1} t_{2} t_{3}\left(t_{1} t_{2}-t_{2} t_{3}^{2}+t_{3}^{2}\right), \quad \Delta_{2}\left(L^{\prime}\right)=1 .
\end{aligned}
$$


Either case shows that $L \not \equiv L^{\prime}$.

It may be interesting to remark that the maps $D_{i} \mid F$ in $U(F)$ are essentially functions which arise [5], as natural analogues of the Fox derivatives, in the theory of extensions within varieties of Lie algebras. The corresponding maps in the next section are also related to extension theory.

3. Special Jordan algebras. Suppose that 2 is a unit in the ground ring $K$. Consider the previous free associative algebra $\Phi$, and let $\Phi^{+}$ be the Jordan algebra obtained by replacing the multiplication in $\Phi$ by the product $x \circ y=\frac{1}{2}(x y+y x)$. Let $J$ be the corresponding free special Jordan algebra, i.e. the subalgebra of $\Phi^{+}$generated by 1 and $x_{1}, \cdots, x_{n}$ (cf. [6], say).

If $M$ is any Jordan algebra, let $U_{s}(M)$ denote the universal algebra for the special representations of $M$, i.e. the quotient of the tensor algebra with identity on the underlying module of $M$ by the ideal generated by all elements of the form $a \circ b-\frac{1}{2}(a \otimes b+b \otimes a)$ where $a, b \in M$ (cf. [4], or [2], Chapter VII). In particular, by the universal property, $U_{s}(J)=\Phi .^{1}$

Next, if $\pi: M \rightarrow N$ is an epimorphism of special Jordan algebras, let $\pi_{*}: U_{s}(M) \rightarrow U_{s}(N)$ be the induced epimorphism. An argument used in [1, p. 269], for example, shows (essentially) that Ker $\pi_{*}$ is the ideal generated by the image of $\operatorname{Ker} \pi$ under the injection $M \rightarrow U_{s}(M) .{ }^{1}$ Further, if $Y$ is a set of ideal generators for $\operatorname{Ker} \pi$, then the image of $Y$ in $U_{8}(M)$ is a set of ideal generators for $\operatorname{Ker} \pi_{*}$.

In particular, if $N$ is a finitely-generated special Jordan algebra with an epimorphism $\pi: J \rightarrow N$, then $\pi_{*}: U_{s}(J) \rightarrow U_{s}(N)$ defines a presentation of $A=U_{s}(N)$ as a quotient of $\Phi$, and the ideal Ker $\pi_{*}$ is finitely generated if $\operatorname{Ker} \pi$ is. Thus, there exist elementary ideals of $N$ in $A_{c}$ which depend only on the isomorphism type of $N$.

\section{REFERENCES}

1. H. Cartan and S. Eilenberg, Homological algebra, Princeton University Press, Princeton, N. J., 1956.

2. P. M. Cohn, Universal algebra, Harper and Row, New York, 1965.

3. R. H. Fox, Free differential calculus. II, Ann. of Math. 59 (1954), 196-210.

4. N. Jacobson, Structure of alternative and Jordan bimodules, Osaka Math. J. 6 (1954), 1-71.

5. J. Knopfmacher, Extensions in varieties of groups and algebras, Acta Math. 115 (1966), 17-50.

6. L. J. Paige, Jordan algebras, Studies in Modern Algebra, Prentice-Hall, Englewood Cliffs, N. J., 1963.

University of Witwatersrand, Johannesburg, South Africa

${ }^{1}$ Added in proof. See also the forthcoming book by N. Jacobson on Jordan algebras. 